\title{
COMPLETIONS FOR A CLASS OF CONVERGENCE GROUPS
}

\author{
G. D. RICHARDSON
}

ABSTRACr. The purpose of this note is to show that the class of abelian totally bounded regular convergence groups has "regular completions".

For basic definitions and terminology concerning convergence spaces, the reader is asked to refer to [6] and [7]. In a natural way one generalizes the notion of a topological group to a convergence group. For a discussion of the latter concept, we refer the reader to [5], and [4] in the limit space setting.

All groups considered will be abelian, and the Hausdorff property will be included in our use of regularity. An isomorphism from one convergence group into another will be a homeomorphism and a group isomorphism. As in the uniform convergence space setting [3], a convergence group is called totally bounded whenever each ultrafilter is Cauchy.

In noncategory language, a complete regular convergence group $(T, p, \cdot)$ is a regular completion of $(S, q, \cdot)$ provided there is an isomorphism $\phi$ from $(S, q, \cdot)$ onto a dense subset of $(T, p, \cdot)$, which has the property that for any complete regular convergence group $(R, r, *)$ and continuous homomorphism $f$ from $\left(S, q,{ }^{\circ}\right)$ onto $(R, r, *)$, there is an extension $g$ to $(T, p, \cdot)$ such that $g \phi=f$, where $g$ is a continuous homomorphism. As is shown in [2], the latter property is always satisfied for Hausdorff topological completions.

Lemma 1 [8]. Let $A$ be a dense subset of a convergence space $(S, q)$ and let $\mathscr{F}$ be an ultrafilter on $S$. There is an ultrafilter $\mathscr{G}$ on $S$ containing $A$ and such that $\Gamma_{q} \mathscr{G} \leqq \mathscr{F}$ (where $\Gamma_{q} \mathscr{G}$ denotes the filter generated by the $q$-closures of elements from $\mathscr{G})$.

Proposition 2. Let $\left(S, q, \cdot^{\circ}\right)$ be a regular convergence group. Then $q$ and $\pi q$ agree on $q$-Cauchy filters, where $\pi q$ denotes the pretopological modification of $q$.

Proof. Let $\mathscr{F}$ be a $q$-Cauchy filter on $S$ and $\pi q$-converge to $x$. Then $\Gamma_{q} \mathscr{F}^{-1} q$-converges to the identity element and, since $\Gamma_{\alpha} \mathscr{F} \mathscr{F}-1$ is

Received by the editors August 9, 1972 and, in revised form, August 20, 1972.

AMS (MOS) subject classifications (1970). Primary 54A05, 54A20.

(c) American Mathematical Society 1973 
coarser than $\left(\Gamma_{q} \mathscr{F}\right)\left(\Gamma_{q} \mathscr{F}\right)^{-1}, \Gamma_{q} \mathscr{F}$ is $q$-Cauchy. Since $\mathscr{F} \pi q$-converges to $x$, then $\dot{x}$ is finer than $\Gamma_{q} \mathscr{F}$, and so $x$ is an adherent point of $\Gamma_{q} \mathscr{F}$. Hence $\mathscr{F}$ $q$-converges to $x$.

Proposition 3. Let $(S, q, \cdot)$ be a regular, totally bounded convergence group. Then $(S, \pi q, \cdot)$ is a topological group.

Proof. From Proposition 2, $q$ and $\pi q$ agree on ultrafilters. Hence by Theorem 2 of [1], $\pi q \times \pi q=\pi(q \times q)$, and so multiplication is continuous on $(S, \pi q, \cdot)$. We have that $(S, \pi q, \cdot)$ is a convergence group, and by [5] it is a topological group. Since it is $T_{1}$, it is also Hausdorff.

THEOREM 4. Let $(S, q, \cdot)$ be a totally bounded regular convergence group. Then it has a regular completion.

Proof. Let $(T, p, \cdot)$ denote the Hausdorff topological completion of $(S, \pi q, \cdot)$, with isomorphism $\phi$. Define $r$ to be the finest convergence structure on $T$ with the following property: $\Gamma_{n}\left(\mathscr{F}_{1} \mathscr{F}_{2} \cdots \mathscr{F}_{n}\right) r$-converges to $x \in T$, whenever $x=x_{1} x_{2} \cdots x_{n}$, $\phi S$ belongs to each $\mathscr{F}_{k}$, and either $\phi^{-1} \mathscr{F}_{k} q$-converges to $\phi^{-1} x_{k} \in S$ or $\mathscr{F}_{k}$ is an ultrafilter which $p$-converges to $x_{k} \in T-\phi S$.

By definition of $r$, if $\mathscr{F} r$-converges to $x$ then it also $p$-converges to $x$. Further, from Lemma $1, p$ and $r$ agree on ultrafilters and so $\Gamma_{p}=\Gamma_{r}$. Hence $(T, r, \cdot)$ is compact regular and so complete.

Certainly $\phi$ is continuous from $(S, q, \cdot)$ into $(T, r, \cdot)$ and is also a group isomorphism. Supose that $\mathscr{G}$ is a filter on $T$ containing $\phi S$ and $r$-converging to some $x \in \phi S$. Then $\mathscr{G}$ is finer than some $\Gamma_{p}\left(\mathscr{F}_{1} \mathscr{F}_{2} \cdots \mathscr{F}_{n}\right) \cap \dot{x}$. Since each $\mathscr{F}_{k}$ either has the property that $\phi^{-1} \mathscr{F}_{k} q$-converges or $\mathscr{F}_{k}$ is an ultrafilter, then $\phi^{-1} \mathscr{F}_{k}$ is $q$-Cauchy; hence $\phi^{-1} \mathscr{G}$ is $q$-Cauchy. Since $\mathscr{G}$ also $p$ converges to $x, \phi^{-1} \mathscr{G} \pi q$-converges to $\phi^{-1} x$, and so by Proposition 2 $\phi^{-1} \mathscr{G} q$-converges to $x$. Hence $\phi$ is an isomorphism.

The compatability of $(T, \cdot)$ and $r$ follows from the fact that $(T, p, \cdot)$ is a topological group. Finally, let $f$ be a continuous homomorphism from $(S, q, \cdot)$ into a complete regular convergence group $(R, \sigma, *)$; then $\left(\Gamma_{\sigma} f S, \pi \sigma, *\right)$ is a compact Hausdorff topological group. Hence there is a continuous homomorphism $g$ from $(T, p, \cdot)$ into $(R, \pi \sigma, *)$ such that $g \phi=f$, and it follows easily that $g$ is also a continuous homomorphism from $(T, r, \cdot)$ into $(R, \sigma, *)$.

\section{REFERENCES}

1. A. M. Carstens and D. C. Kent, A note on products of convergence spaces, Math. Ann. 182 (1969), 40-44. MR 40 \#3494.

2. N. Bourbaki, General topology, Part I, Hermann, Paris; Addison-Wesley, Reading, Mass., 1966. MR 34 \#5044a. 
3. C. H. Cook and H. R. Fischer, Uniform convergence structures, Math. Ann. 173 (1967), 290-306. MR 36 \#845.

4. H. R. Fischer, Limesräume, Math. Ann. 137 (1959), 269-303. MR 22 \#225.

5. D. C. Kent, On convergence groups and convergence uniformities, Fund. Math. 60 (1967), 213-222. MR 36 \#842.

6. ——, Convergence quotient maps, Fund. Math. 65 (1969), 197-205. MR 40 \#3497.

7. D. C. Kent and G. D. Richardson, Minimal convergence spaces, Trans. Amer. Math. Soc. 160 (1971), 487-499. MR 44 \#3279.

8. - Regular compactifications of convergence spaces, Proc. Amer. Math. Soc. 31 (1972), 571-573. MR 44 \#3290.

Department of Mathematics, East Carolina University, Greenville, North Carolina 27834 